\title{
Bibliografia Russa Traduzida no Brasil (1900-1950)
}

\author{
Denise Bottmann ${ }^{1}$
}

Abstract: This survey provides extensive bibliographical data on Russian literary works which have been translated into Portuguese and published in Brazil in the first half of twentieth century. It has as its purpose to be useful as a sort of archive for ulterior researchers, who will be able to find here many primary data which were until now scattered among several sources.

Keywords: Russian literature; Brazilian translations; history of translation in Brazil

Resumo: Este levantamento abrange uma extensa bibliografia de obras literárias russas traduzidas e publicadas no Brasil na primeira metade do século XX. Seu objetivo é fornecer uma base de dados para pesquisadores da área, servindo como compilação consolidada de informações primárias até então dispersas em várias fontes.

Palavras-chave: Literatura russa; traduções brasileiras; história da tradução no Brasil

\section{Introdução}

O presente levantamento arrola obras literárias de autores russos e soviéticos que foram publicadas em livro no Brasil entre 1900 e 1950. Como ponto de partida, tomei a inestimável listagem de edições e traduções brasileiras em periódicos e livros publicados entre 1887 e 1936, apresentada por Bruno Gomide em sua tese de doutorado (2004, 485-488).

Embora este mapeamento tenha pretensões de ser razoavelmente exaustivo, sem dúvida não passará isento de lacunas. Por ora, aqui estão reunidos 81 autores, em 222 livros publicados, abrangendo cerca de 470 textos entre contos, novelas e romances. Foram incluídos alguns textos não literários, desde que guardassem alguma relação, pelo tema ou pelo autor, com os objetivos do levantamento.

A pesquisa não se deteve sobre a língua de partida para as traduções; conste apenas que uma pequeníssima parcela dos textos foi traduzida diretamente do original por Ivan Emilianovitch, Georges Selzoff, Evandro Pequeno e Boris Schnaiderman (assinando como Boris Solomonov) e talvez algum outro. Na maioria dos casos, o idioma usado na interposição foi o francês, mas há também traduções feitas a partir do espanhol e, em menor medida, do inglês.

\footnotetext{
${ }^{1}$ Mestre, docente IFCH/UNICAMP (1983-1998), historiadora e tradutora. dbottmann@gmail.com.
} 
Nem todas as edições apresentam os responsáveis pelas traduções, as quais ficam aqui registradas como anônimas; em alguns outros casos, simplesmente não consegui rastrear a identidade do tradutor. Ademais, há vários casos de editoras que republicam traduções antes lançadas por outras editoras, tanto brasileiras quanto portuguesas, sem fornecer referências ou apenas indicando que se trata de "uma tradução revista" por algum colaborador da casa.

A apresentação está disposta em ordem alfabética por autor, cujo nome vem grafado em negrito em sua padronização atual; entre parênteses, constam as outras grafias encontradas nas diversas edições. Nas entradas individuais por autor, as obras também estão dispostas em ordem alfabética. Salvo outra indicação, as datas registradas correspondem ao ano da primeira edição.

\section{Bibliografia 1900-1950}

Andréiev, Leonid (Leonide, Leônidas, Andreev, Andreieff):

“A conversão do Diabo". In: As obras-primas do conto universal. Trad. Almiro R. Barbosa e Edgard Cavalheiro. Porto Alegre: Martins, 1943.

A gargalhada vermelha. Trad. B. Seibel. Coleção Seleta, vol. 6. São Paulo: Assunção, c.1945. Reed. São Paulo: Anchieta, 1946.

Judas Iscariotes. Trad. anôn. [Georges Selzoff e prov. Fúlvio Abramo]. Bibliotheca dos Auctores Russos. ${ }^{2}$ São Paulo: Cultura, 1931.

Judas Iscariotes. Trad. anôn. Edições Colête. São Paulo: A Bolsa do Livro, 1944.

O diário de Satanás. Trad. Elias Davidovitch. Rio de Janeiro: Renascença, 1933.

Os sete enforcados. Trad. Georges Selzoff e Orígenes Lessa. Bibliotheca dos Auctores Russos. São Paulo: Cultura, 1931. Reed. in: Os russos: antigos e modernos [porém indicando apenas Lessa nos créditos de tradução]. Rio de Janeiro: Leitura, 1944.

“Os sete enforcados". In: 3 novelas russas. Trad. José de Barros Pinto. São Paulo: Flama, 1944.

“Os sete enforcados". In: Três novelas russas. "Tradução revista pelo departamento editorial" [versão adulterada da tradução de Selzoff/ Lessa, Cultura, 1931]. Coleção Grandes Romances Universais. São Paulo: W. M. Jackson, 1947.

"Por trás da janela". In: Os colossos do conto da velha e da nova Rússia. Trad. Manuel R. da Silva. Rio de Janeiro: Mundo Latino, 1944.

\footnotetext{
${ }^{2}$ Para uma crônica de Georges Selzoff e sua "Bibliotheca dos Auctores Russos", ver Bottmann (2013/1).
} 
"Silêncio". In: Os mais belos contos russos dos mais famosos autores. 1ª Série. Trad. Enéias Marzano. Rio de Janeiro: Vecchi, 1945.

“Uma flor espezinhada". In: Os mais belos contos russos dos mais famosos autores. $2^{\mathrm{a}}$. série. Trad. Alfredo Ferreira. Rio de Janeiro: Vecchi, 1945.

\section{Ardov, Victor:}

"O papão". In: Os mais belos contos burlescos, irônicos e sarcásticos, dos mais famosos autores. Trad. Alfredo Ferreira. Rio de Janeiro: Vecchi, 1947.

Artzybachev, Mikhail P. (Mikail, Artzibachev):

“O revolucionário”. In: Os russos: antigos e modernos. Trad. Esther Mesquita. Coleção Contos do Mundo. Rio de Janeiro: Leitura, 1944.

“O velho procurador narrou”. In: Os colossos do conto da velha e da nova Rússia. Trad. Gilberto Galvão. Rio de Janeiro: Mundo Latino, 1944.

"Um assassinato". In: Os mais belos contos russos dos mais famosos autores. 1". Série. Trad. Manuel R. da Silva. Rio de Janeiro: Vecchi, 1945.

Averchenko, Arkadi (Arkadio, Arcádio, Averchenko):

“A maldição da glória”. In: Os mais belos contos russos dos mais famosos autores. 2 a série. Trad. Alfredo Ferreira. Rio de Janeiro: Vecchi, 1945.

"A odisséia de uma vaca". In: Contos soviéticos. Trad. Luiz Alípio de Barros. Rio de Janeiro: O Cruzeiro, 1944.

“Oedipo Rei”. In Antologia dos grandes contos humorísticos. Trad. anôn. São Paulo: Brasiliense, 1944.

“Um drama sensacional”. In: Os colossos do conto da velha e da nova Rússia. Trad. Manuel R. da Silva. Rio de Janeiro: Mundo Latino, 1944.

“Uma vaca!”. In: Os mais belos contos russos dos mais famosos autores. 1. Série. Trad. Frederico dos Reys Coutinho. Rio de Janeiro: Vecchi, 1945.

\section{Babel, Isaac:}


Cavalaria vermelha. Trad. Jorge Amado. ${ }^{3}$ Coleção Ontem e Hoje, vol. 16. São Paulo: Brasiliense, 1945.

“Depois da luta". In: Contos soviéticos. Os novos da Rússia. Trad. Gabriel Marques. Collecção Literatura Moderna. São Paulo: Cultura Brasileira, c.1934. Reed. in: Contos soviéticos. Rio de Janeiro: O Cruzeiro, 1944.

"O despertar". In: Os russos: antigos e modernos. Trad. Moacir Werneck de Castro. Coleção Contos do Mundo. Rio de Janeiro: Leitura, 1944.

"Rabi". In: Os colossos do conto da velha e da nova Rússia. Trad. Frederico dos Reys Coutinho. Rio de Janeiro: Mundo Latino, 1944.

Bashkirtseva, Maria Konstantinova (Marie, Bashkirtseff):

Diário. Trad. Gilda Marinho. Coleção Nobel. Porto Alegre Globo, 1943.

\section{Berkovitch, Itzoc-Dob:}

"Sob a ameaça do Pogrom". In: Os mais belos contos russos dos mais famosos autores. $2^{\mathrm{a}}$. série. Trad. Gilberto Galvão. Rio de Janeiro: Vecchi, 1945.

Berzin, Julij S. (Julio):

$100 \%$ de amor, especulação e volupia (amor e especulação no paiz dos soviets). Trad. Zoran Ninitch. Rio de Janeiro: Marisa, c.1934.

\section{Bierdiáiev, Nikolai (Berdiaev):}

O espírito de Dostoievski. Trad. Otto Schneider. Rio de Janeiro: Panamericana, c.1944.

Uma nova Idade Média. Trad. Tasso da Silveira. Rio de Janeiro: José Olympio, 1936.

Bukhov, Arkadi S. (Bourkhov):

"Fazer a côrte". In: Os mais belos contos russos dos mais famosos autores. $2^{\mathrm{a}}$. série. Trad. J. da Cunha Borges. Rio de Janeiro: Vecchi, 1945.

"O fim de Sherlock Holmes". In: Contos soviéticos. Trad. Luiz Alípio de Barros. Rio de Janeiro: O Cruzeiro, 1944.

\footnotetext{
${ }^{3}$ Embora Jorge Amado conste como tradutor desta e de outras sete obras presentes neste levantamento, ele apenas emprestou seu nome aos créditos de tradução na Coleção Ontem e Hoje, pela Brasiliense, como uma espécie de chancela de esquerda e chamariz para os leitores.
} 
"Regras de conduta para uso alheio". In: Os mais belos contos russos dos mais famosos autores. $1^{\text {a }}$. Série. Trad. Frederico dos Reys Coutinho. Rio de Janeiro: Vecchi, 1945.

\section{Bunin, Ivan:}

"A floresta". In: Os colossos do conto da velha e da nova Rússia. Trad. Frederico dos Reys Coutinho. Rio de Janeiro: Mundo Latino, 1944.

"A glória". In: Os mais belos contos russos dos mais famosos autores. $2^{\mathrm{a}}$. série. Trad. Frederico dos Reys Coutinho. Rio de Janeiro: Vecchi, 1945.

A noite. Trad. Zoran Ninitch. Rio de Janeiro: Calvino, 1934.

O amor de Mitia. Trad. Zoran Ninitch. Rio de Janeiro: Guanabara, 1933.

“O cavalheiro de São Francisco". In: Os russos: antigos e modernos. Trad. Jorge de Lima. Coleção Contos do Mundo. Rio de Janeiro: Leitura, 1944.

O senhor de São Francisco. Trad. Zoran Ninitch. Rio de Janeiro: Mundial, c.1934.

Chchedrin, Nicolai (Nicolau; pseud. de Mikhail Saltikhov-Schedrin):

"Dois pequenos mujiques". In: Os russos: antigos e modernos. Trad. Maria Julieta e Carlos Drummond de Andrade. Coleção Contos do Mundo. Rio de Janeiro: Leitura, 1944.

"Um mujique alimenta dois funcionários públicos”. In: Os russos: antigos e modernos. Trad. Rubem Braga. Coleção Contos do Mundo. Rio de Janeiro: Leitura, 1944.

Cholokhov, Mikhail (Mikail, Sholokhov, Skholotchof, Scholochov, Cholokov):

O Don silencioso. Trad. Ligia Junqueira e, na parte poética, Agnaldo Junqueira Filho. Rio de Janeiro: O Cruzeiro, 1945.

“O pae”. In: Contos soviéticos. Os novos da Rússia. Trad. Gabriel Marques. Collecção Literatura Moderna. São Paulo: Cultura Brasileira, c.1934. Reed. in: Contos soviéticos. Rio de Janeiro: O Cruzeiro, 1944.

"Os grandes pelos pequenos". In: Os mais belos contos russos dos mais famosos autores. $1^{\mathrm{a}}$. Série. Trad. Manuel R. da Silva. Rio de Janeiro: Vecchi, 1945.

Terra e sangue. Trad. Gustavo Nonnenberg. São Paulo: Flama, 1945.

Chpanov, Nikolai (R., Shpanov):

“O homem de óculos". In: Os mais belos contos russos dos mais famosos autores. $2^{\mathrm{a}}$. série. Trad. Alfredo Ferreira. Rio de Janeiro: Vecchi, 1945. 
Dimov, Ossip (Osip, Dimof):

"A falência". In: Os mais belos contos russos dos mais famosos autores. 1ª Série. Trad. Marina Salles Goulart de Andrade. Rio de Janeiro: Vecchi, 1945.

"Bancarrota". In: Contos soviéticos. Os novos da Rússia. Trad. Gabriel Marques. Collecção Literatura Moderna. São Paulo: Cultura Brasileira, c.1934. Reed. in: Contos soviéticos. Rio de Janeiro: O Cruzeiro, 1944.

\section{Dorochevitch, Vlas (Vlass):}

“Assim falou Tchi-San”. In: Contos soviéticos. Trad. Luiz Alípio de Barros. Rio de Janeiro: O Cruzeiro, 1944.

“Os calcanhares são outros". In: Os mais belos contos russos dos mais famosos autores. 1ª Série. Trad. Frederico dos Reys Coutinho. Rio de Janeiro: Vecchi, 1945.

Dostoiévski, Fiódor M. (Fédor, Fiodor, Theodore, Teodoro, Dostoevsky, Dostoiewsky):

“A árvore de Cristo". In: Livro de Natal - As mais lindas histórias de Natal dos maiores escritores do mundo. Trad. anôn. São Paulo: Martins, 1947.

"A mulher do outro - aventura extraordinária". In: Três novelas russas. Trad. Lúcio Cardoso. Rio de Janeiro: A Noite, 1950.

A órfã. Trad. Adolfo Bezerra de Menezes Neto. Rio de Janeiro: Edições do Povo, 1947. Alma de creança. Trad. Henrique Marques Junior. Collecção Chic. Rio de Janeiro: Azevedo \& Costa, 1915. Reed. Alma de criança. São Paulo: Brasileira, 1936.

Alma de creança. Trad. anôn. Rio de Janeiro: Universal, 1932.

Alma de creança. Trad. anôn. Collecções Econômicas SIP, vol. 42. Rio de Janeiro: Civilização Brasileira, 1936.

“Bobok (Recordações de alguém)". Trad. Frederico dos Reys Coutinho. In: Os colossos do conto da velha e da nova Rússia. Rio de Janeiro: Mundo Latino, 1944.

Crime e Castigo. Trad. Fernão Neves (pseud. de Fernando Nery). Rio de Janeiro, Castilho, c. 1916. 
Crime e castigo. Trad. Ivan Petrovitch. Collecção de Obras Celebres. ${ }^{4}$ Rio de Janeiro: Americana, 1930. Reed. com trad. revista por Elias Davidovitch. Rio de Janeiro: Guanabara, 1936.

Crime e castigo. Trad. J. Jobinski, revista por Aurélio Pinheiro. Rio de Janeiro: Pongetti, 1936. Reed. em trad. revista por Marques Rebelo pela mesma Pongetti em 1941.

Crime e castigo. Trad. anôn. Série O Livro de Bolso. São Paulo: O Livro de Bolso, 1941.

Crime e castigo. Trad. anôn. ["Tradução da Editora"]. São Paulo: Cruzeiro do Sul, 1943 ( $2^{\mathrm{a}}$. ed.).

Crime e castigo. Trad. Rosário Fusco. Coleção Fogos Cruzados. Rio de Janeiro: José Olympio, 1949.

Diário de um escritor. Trad. Frederico dos Reys Coutinho. Rio de Janeiro: Vecchi, 1943.

"Ela era doce e humilde". In: Os russos: antigos e modernos. Trad. Valdemar Cavalcanti. Coleção Contos do Mundo. Rio de Janeiro: Leitura, 1944.

Ensaio sobre o burguez. Trad. Elias Davidovitch. Rio de Janeiro: Minha Livraria, c. 1934.

Humilhados e ofendidos. Rio de Janeiro: Civilização Brasileira, 1935.

Humilhados e ofendidos. Trad. Rachel de Queiroz. Rio de Janeiro: José Olympio, 1944.

Humilhados e offendidos. Trad. revista por Bandeira Duarte. Rio de Janeiro: Marisa, 1931.

Netotchka. Trad. Costa Neves. Rio de Janeiro: Brasil, 1937. Reed. como Nietotchka Niezvanova. Rio de Janeiro: José Olympio, 1947.

Noites brancas, Está morta, O grande inquisidor. Trad. anôn. São Paulo: Clube do Livro, 1948. ${ }^{5}$

O embusteiro. Trad. Carlos M. A. Bittencourt. São Paulo: Assunção, 1945.

O espírito subterrâneo. Trad. Rosário Fusco. Rio de Janeiro: Panamericana, c.1944.

O eterno marido. Trad. Violeta Alcântara Carreira. São Paulo: Cultura Brasileira, 1935.

Reed. São Paulo: Martins, 1944.

\footnotetext{
${ }^{4}$ O catálogo literário da Americana passou a fazer parte, em 1931, da Waissman, Reis \& Cia. (a qual, por sua vez, adquire a Guanabara em 1934, adotando-lhe o nome). Daí a Collecção de Obras Celebres presente nas duas editoras, como se verá em outros títulos adiante.

${ }^{5}$ Salvo algumas exceções, o Clube do Livro utilizava sem licença traduções lançadas por outras editoras, sobretudo portuguesas, publicando-as anonimamente ou atribuindo-as a um colaborador da casa, José Maria Machado.
} 
O eterno marido. Trad. Costa Neves. [Edição especial para bibliófilos, com tiragem restrita de 200 exemplares.] Rio de Janeiro: José Olympio, $1944 .^{6}$

O eterno marido. Trad. anôn. São Paulo: Clube do Livro, 1946.

O idiota. Trad. José Geraldo Vieira. Rio de Janeiro: José Olympio, 1949.

O jogador. Trad. Otto Schneider. Rio de Janeiro: Panamericana, 1943.

O jogador. Trad. anôn. São Paulo: Clube do Livro, 1945.

O príncipe idiota. Trad. Demerval Café e Oswaldo Castro. Rio de Janeiro: Waissman, Reis \& Cia., 1931.

"O sonho de um homem ridículo". In: Contos russos. Trad. anôn. Edições Colête, vol. 7. São Paulo: A Bolsa do Livro, 1944.

"O sonho de um homem ridículo (Conto fantástico)". In: Os mais belos contos russos dos mais famosos autores. $2^{\mathrm{a}}$. série. Trad. Frederico dos Reys Coutinho. Rio de Janeiro: Vecchi, 1945.

O sonho do tio. Trad. anôn. Edições Colête. São Paulo: A Bolsa do Livro, 1945.

O sósia. Trad. Corália Rego Lins. Rio de Janeiro: Vecchi, 1943.

O tyrano. Trad. Elias Davidovitch. Rio de Janeiro: Calvino, 1933.

Os irmãos Karamazoff. Trad. Raul Rizinsky. Collecção de Obras Celebres. Rio de Janeiro: Americana, 1931.

Os irmãos Karamazov. Trad. Paulo Mendes de Almeida. São Paulo: Martins, 1944.

Os irmãos Karamazov. Trad. Boris Solomonov (pseud. de Boris Schnaiderman). Coleção As Obras Eternas. Rio de Janeiro: Vecchi, 1944.

Os pobres diabos. Trad. Elias Davidovitch. Rio de Janeiro: Flores e Mano, 1932.

Os possessos. Trad. Augusto Rodrigues. Rio de Janeiro: Panamericana, c.1943.

Recordação da casa dos mortos. Trad. Fernão Neves (pseud. de Fernando Nery). Rio de Janeiro: Castilho, 1917.

Recordações da casa dos mortos. Trad. Antônio de Oliveira Garcia. Coleção Excelsior. São Paulo: Martins, 1942.

Recordações da casa dos mortos. Trad. revista por Joaquim Moura de Menezes. São Paulo: Cia. Brasil, c. 1945.

Recordações da casa dos mortos. Trad. Rachel de Queiroz. Rio de Janeiro: José Olympio, 1945.

\footnotetext{
${ }^{6}$ Apenas em 1951 a José Olympio relançará essa tradução, agora em edição normal, em sua Coleção Fogos Cruzados, vol. 40.
} 
Recordações da casa dos mortos. Trad. José Geraldo Vieira. Coleção Saraiva, vol. 7. São Paulo: Saraiva, 1948.

Stepantchikovo. Trad. D. Martins de Oliveira. Rio de Janeiro: Século XX, 1942.

Um jogador (das notas de um rapaz) - Igrok. Trad. anôn. [Georges Selzoff e Allyrio M. Wanderley]. Bibliotheca de Auctores Russos. São Paulo: Cultura, 1931.

Um jogador (notas de um jovem). Trad. Costa Neves. Rio de Janeiro: José Olympio, 1944.

"Um ladrão honrado". In: Os mais belos contos russos dos mais famosos autores. 1". Série. Trad. Manuel R. da Silva. Rio de Janeiro: Vecchi, 1945.

Ehrenburg, Ilya (Elias, Ilia, Ehrenbourg):

A epopéia russa. Diário de um jornalista junto ao Exército Vermelho. Trad. Maslowa Gomes Venturi. Coleção A Marcha do Tempo, vol. 7. São Paulo: Brasiliense, 1946.

A queda de Paris. Trad. Monteiro Lobato. Coleção Guerra e Paz, vol. 4. São Paulo: Nacional, 1944.

As aventuras de Julio Jurenito. Trad. Mauro Rosalvo. Rio de Janeiro: Civilização Brasileira, 1932.

Fevereiro sangrento - a Revolução de 1934 na Áustria. Trad. Carlos Lacerda. Rio de Janeiro: Alba, c. 1935.

"História de um cachimbo de barro". In: Os mais belos contos russos dos mais famosos autores. $2^{\mathrm{a}}$. série. Trad. Líbero Rangel de Andrade. Rio de Janeiro: Vecchi, 1945.

Morte ao invasor alemão. Trad. Moise Wainer. Rio de Janeiro: Vitória, 1944.

“O bar "Ao Descanso Vermelho"”. In: Contos soviéticos. Os novos da Rússia. Trad. Gabriel Marques. Collecção Literatura Moderna. São Paulo: Cultura Brasileira, c.1934. Reed. in: Contos soviéticos. Rio de Janeiro: O Cruzeiro, 1944.

“O cachimbo de Josué”. In: Os mais belos contos russos dos mais famosos autores. $1^{\text {a }}$. Série. Trad. Manuel R. da Silva. Rio de Janeiro: Vecchi, 1945.

"O cachimbo do dr. Peterson". In: Os colossos do conto da velha e da nova Rússia. Trad. Líbero Rangel de Andrade. Rio de Janeiro: Mundo Latino, 1944.

O degêlo. Trad. David Medeiros. Rio de Janeiro: Vitória, 1944.

O segundo dia da criação. Trad. Alfredo Ferreira. Coleção Eros. São Paulo: Prometeu, 1946.

"Três cachimbos". In: Os russos: antigos e modernos. Trad. Osório Borba. Coleção Contos do Mundo. Rio de Janeiro: Leitura, 1944. 
Treze cachimbos. Trad. David Medeiros. Rio de Janeiro: Vitória, 1944.

\section{Fadeiev, Aleksandr A.:}

A derrota. Trad. Helio de Andrade (pseud. de Leôncio Basbaum). São Paulo: Urania, 1931.

Fedin, Konstantin (Constantino, Fedine):

“Alma de cachorro". In: Contos soviéticos. Os novos da Rússia. Trad. Gabriel Marques. Collecção Literatura Moderna. São Paulo: Cultura Brasileira, c.1934. Reed. in: Contos soviéticos. Rio de Janeiro: O Cruzeiro, 1944.

As cidades e os anos. Trad. Humberto Schoenfeldt e Augusto de Sousa. São Paulo: Ipê, 1947.

O sanatório do doutor Klebe. Trad. Jorge Amado [vide nota 3]. Coleção Ontem e Hoje, vol. 13. São Paulo: Brasiliense, 1945.

"Um homem resoluto". In: Os colossos do conto da velha e da nova Rússia. Trad. Manuel R. da Silva. Rio de Janeiro: Mundo Latino, 1944.

\section{Fibitch, Daniel:}

Os libertos. Trad. Zoran Ninitch. Coleção Aurora. São Paulo: Unitas, 1934.

\section{Garin, Nicolai (Nicolas):}

"A primavera da vida". In: Três novelas russas. Trad. Lúcio Cardoso. Rio de Janeiro: A Noite, 1950.

"U'a mulher esquisita". In: Os mais belos contos russos dos mais famosos autores. $1^{\mathrm{a}}$. Série. Trad. Marina Salles Goulart de Andrade. Rio de Janeiro: Vecchi, 1945.

\section{Garshin, Vsevolod M. (Garchin):}

“De propósito não foi”. In: Os mais belos contos russos dos mais famosos autores. 2a série. Trad. Alfredo Ferreira. Rio de Janeiro: Vecchi, 1945.

"O sinal”. In: Os russos: antigos e modernos. Trad. Lourival Gomes Machado. Coleção Contos do Mundo. Rio de Janeiro: Leitura, 1944.

Gladkov, Fédor (Fiodor, Gladkof):

Cimento. Trad. anôn. Coleção Aurora. São Paulo: Unitas, 1933. 
“O polvo". In: Contos soviéticos. Os novos da Rússia. Trad. Gabriel Marques. Collecção Literatura Moderna. São Paulo: Cultura Brasileira, c.1934. Reed. in: Contos soviéticos. Rio de Janeiro: O Cruzeiro, 1944.

Gogol, Nikolai (Nicolau, Nicolas):

"A carta extraviada". In: Os colossos do conto da velha e da nova Rússia. Trad. Frederico dos Reys Coutinho. Rio de Janeiro: Mundo Latino, 1944. Reed. in: Os mais belos contos russos dos mais famosos autores. Segunda Série. Rio de Janeiro: Vecchi, 1945.

Almas mortas - Aventura de Chichicov. Trad. Costa Neves. Rio de Janeiro: Cia. Brasil, 1937. Reed. como Almas mortas - As aventuras de Chichicov (poemas em prosa). Coleção Fogos Cruzados. Rio de Janeiro: José Olympio, 1941.

"Memórias de um louco". In: Os mais belos contos russos dos mais famosos autores. $1^{\text {a }}$. Série. Trad. Marina Salles Goulart de Andrade. Rio de Janeiro: Vecchi, 1945.

"Noite de São João". In: Os colossos do conto da velha e da nova Rússia. Trad. Edison Carneiro. Rio de Janeiro: Mundo Latino, 1944.

"O capote". In: Os russos: antigos e modernos. Trad. Vinicius de Moraes [e um cotradutor não nomeado, proficiente no idioma russo]. Coleção Contos do Mundo. Rio de Janeiro: Leitura, 1944.

O inspetor: comédia em 5 atos. Trad. Zygmunt Turkow. Rio de Janeiro: Leitura, 1945.

"O nariz". In: Antologia dos grandes contos humorísticos. Trad. anôn. São Paulo: Brasiliense, 1944.

Tarass Boulba. Trad. A. Tenório d'Albuquerque. Rio de Janeiro: Cia. Brasil, 1937.

“Tarass Bulba". In: Três novelas russas. "Tradução revista pelo departamento editorial”. Coleção Grandes Romances Universais. São Paulo: W. M. Jackson, 1947.

\section{Goomilevski, Léo:}

O amor em liberdade. Trad. revista por Galeão Coutinho. Collecção Literatura Moderna. São Paulo: Cultura Brasileira, c.1934. Reed. Rio de Janeiro: O Cruzeiro, 1944.

Górki, Maksim (Máximo, Maxime, Maxim, Gorki, Gorky):

A infância de Klim Sanguine. Trad. Lúcio do Nascimento Rangel. Rio de Janeiro: Athena, 1937. 
A mãe. Rio de Janeiro: Marisa, 1931.

A mãe. Trad. revista por Renato Travassos. Collecção de Obras Celebres. Rio de Janeiro: Americana, 1931.

A mãe. Rio de Janeiro: Calvino, 1932.

A mãe. Collecções Econômicas SIP. Rio de Janeiro: Civilização Brasileira, 1935.

A mãe. Rio de Janeiro: Vitória, 1944.

A mãe. Trad. Araújo Neves. Rio de Janeiro: Pongetti, 1944.

A minha infancia. Rio de Janeiro: Minha Livraria, c.1934.

"Adversários". In: O espírito do conto francês, alemão, ianque, inglês, russo. Trad.

Sonia Moniz. Coleção Cultura Moderna. Rio de Janeiro: Curiosidade, 1942.

“Angústia". In: Os colossos do conto da velha e da nova Rússia. Trad. Frederico dos Reys Coutinho. Rio de Janeiro: Mundo Latino, 1944.

Aos intelectuais. Carta aberta (em resposta a alguns escritores americanos). Rio de Janeiro: Alba, c. 1935.

Contos da Estépe. Contém: "Impressões de um homem pacífico", "Os três", "O avô Arkhip e Lenka", "O canto do falcão", "Yemelian Pilaie", "O Khan e seu filho", "Sasubrina", "Makar Tchudra", "Vinte seis e uma" e "A velha Iserguila". Trad. revista por Antônio de Oliveira. Rio de Janeiro: Nosso Livro, $1944 .^{7}$

Em guarda! Aspectos da Rússia soviética. Rio de Janeiro: Adersen, 1934

Flor da miséria e outras histórias. Rio de Janeiro: Edições do Povo, $1947 .{ }^{8}$

Ganhando meu pão: memórias autobiográficas. Trad. Abelardo Romero. Rio de Janeiro: Vecchi, 1942.

“Kirilka". In: Os russos: antigos e modernos. Trad. Murilo Miranda. Coleção Contos do Mundo. Rio de Janeiro: Leitura, 1944.

Konovaloff. Contém: "Konovaloff”, “A feira", "Kirilka” e "Uma vez, no outomno". Trad. anôn. [Georges Selzoff e F. Olandim]. Bibliotheca de Auctores Russos. São Paulo: Cultura, 1930.

Lenine. Rio de Janeiro: Minha Livraria, c.1934.

Na prisão. Trad. Djalma Maciel. Rio de Janeiro: Edições do Povo, 1946.

O espião. Trad. revista por Bandeira Duarte. Rio de Janeiro: Marisa, 1931.

\footnotetext{
7 Como "O avô Arkhip e Lenka", "O canto do falcão", "O Khan e seu filho" e "Makar Tchudra" tinham sido publicados em 1927 e 1928 no periódico Primeira: a revista por excelência, em tradução anônima (GOMIDE, 2004, 408), não excluo a hipótese de que essa antologia de Contos da estépe seja uma compilação de publicações anteriores.

${ }^{8}$ Foram baldados os esforços em ter acesso ao conteúdo desta pequena coletânea (73 páginas).
} 
O espião. Rio de Janeiro: Civilização Brasileira, 1934.

O espião. Trad. Milton Tavares. Rio de Janeiro: Vitória, 1944.

“O herói”. In: Os mais belos contos russos dos mais famosos autores. $2^{\mathrm{a}}$. série. Trad.

Líbero Rangel de Andrade. Rio de Janeiro: Vecchi, 1945.

“O ruivo”. In: 3 Novelas russas. Trad. José de Barros Pinto. São Paulo: Flama, 1944.

Os Artamonov. Trad. Boris Solomonov (pseud. de Boris Schnaiderman) e Galvão de Queiroz. Coleção “Os Grandes Nomes”. Rio de Janeiro: Vecchi, 1945.

Os degenerados: os Orlof, os ex-homens, os amigos. São Paulo: Editorial Paulista, 1934.

Os degenerados. Rio de Janeiro: Civilização Brasileira, 1936.

Os degenerados. Trad. revista por Marques Rebelo. Rio de Janeiro: Pongetti, 1943.

Os vagabundos. Collecções Econômicas SIP. Rio de Janeiro: Civilização Brasileira, 1936.

Os vagabundos. Trad. Araújo Alves. Rio de Janeiro: Pongetti, 1944.

Psychologia do povo russo. Trad. Elias Davidovitch. Rio de Janeiro: Minha Livraria, 1936.

"Sonho de uma noite de Natal". In: Livro de Natal - As mais lindas histórias de Natal dos maiores escritores do mundo. Trad. Luiz Macedo. São Paulo: Martins, 1947.

Tormenta sobre a cidade. Trad. J. da Cunha Borges. Rio de Janeiro: Vecchi, 1943.

Três russos: Tolstoi, Tchekov, Andreev. Trad. Lúcio do Nascimento Rangel. Coleção Pensamento e Vida. Rio de Janeiro: Pongetti, 1945.

"Um incidente". In: Os mais belos contos russos dos mais famosos autores. 1". Série. Trad. Marina Salles Goulart de Andrade. Rio de Janeiro: Vecchi, 1945.

Uma confissão. Collecção de Obras Celebres. Rio de Janeiro: Waissman, Reis \& Cia., 1931.

Uma confissão. Rio de Janeiro: Calvino, 1932.

“Uma noite de outono". In: Os russos: antigos e modernos. Trad. Emil Farhat. Coleção

Contos do Mundo. Rio de Janeiro: Leitura, 1944.

Varenka Olessova. Trad. anôn. São Paulo: Clube do Livro, 1949.

"Vinte e seis e uma". In: Os russos: antigos e modernos. Trad. Edison Carneiro.

Coleção Contos do Mundo. Rio de Janeiro: Leitura, 1944.

Wania. Trad. anôn. São Paulo: Unitas, 1934.

\section{Grossman, Vassili:}


Povo imortal. Trad. Godin Sampaio Vianna. São Paulo: Renascença, 1945.

\section{Ilf, Ilya; Petrof, Eugen.}

A Aventura das doze cadeiras. Trad. Milton Tavares. Rio de Janeiro: Vitória, 1944.

Ilin, Mikhail (pseud. de Ilia Iakovlevich Marshak):

Preto no branco. Trad. Josué Almeida. Rio de Janeiro: Vitória, 1944.

Ivanov, Vsevolod (Ivanof, Wsievolof):

"A criança". In: Os russos: antigos e modernos. Trad. Dalcídio Jurandir. Coleção Contos do Mundo. Rio de Janeiro: Leitura, 1944.

"O menino". In: Contos soviéticos. Os novos da Rússia. Trad. Gabriel Marques. Collecção Literatura Moderna. São Paulo: Cultura Brasileira, c.1934. Reed. in: Contos soviéticos. Rio de Janeiro: O Cruzeiro, 1944.

O trem blindado 14-69. Trad. Jorge Amado [vide nota 3]. Coleção Ontem e Hoje, vol. 11. São Paulo: Brasiliense, 1945.

\section{Kalinikov, Joseph:}

A tragédia sexual de Leon Tolstoi. Trad. Zoran Ninitch. Rio de Janeiro: Marisa, 1934.

\section{Katayev, Valentine:}

“As facas". In: Os russos: antigos e modernos. Trad. Elsie Lessa. Coleção Contos do Mundo. Rio de Janeiro: Leitura, 1944.

Kolontai, Aleksandra (Alexandra):

“Irmãs”. In: Contos soviéticos. Os novos da Rússia. Trad. Gabriel Marques. Collecção Literatura Moderna. São Paulo: Cultura Brasileira, c.1934. Reed. in: Contos soviéticos. Rio de Janeiro: O Cruzeiro, 1944.

\section{Korolenko, Vladimir (Vladimiro):}

“O sonho de Makar”. In: Os colossos do conto da velha e da nova Rússia. Trad. José Dauster. Rio de Janeiro: Mundo Latino, 1944.

“O velho sineiro". In: Os mais belos contos russos dos mais famosos autores. $2^{\mathrm{a}}$. série. Trad. Enéias Marzano. Rio de Janeiro: Vecchi, 1945. 
“Os murmúrios da selva". In: Os mais belos contos russos dos mais famosos autores. 1". Série. Trad. Marina Salles Goulart de Andrade. Rio de Janeiro: Vecchi, 1945.

“Uma rapariga estranha”. In: Os russos: antigos e modernos. Trad. Aurélio Buarque de Hollanda. Coleção Contos do Mundo. Rio de Janeiro: Leitura, 1944.

\section{Kozhevnikov, Vadim:}

"Março e abrill". In: Os russos: antigos e modernos. Trad. Fritz Teixeira Sales. Coleção Contos do Mundo. Rio de Janeiro: Leitura, 1944.

Kuprin, Aleksandr I. (Alexandre, Alexander, Kouprine):

A fossa. Trad. Boris Solomonov (pseud. de Boris Schnaiderman). Rio de Janeiro: Panamericana, 1944.

“A pulseira de rubis". In: Os mais belos contos russos dos mais famosos autores. $1^{\mathrm{a}}$. Série. Trad. Gama e Silva. Rio de Janeiro: Vecchi, 1945.

"Lenotchka". In: Os russos: antigos e modernos. Trad. Lia Corrêa Dutra. Coleção Contos do Mundo. Rio de Janeiro: Leitura, 1944.

"Natália Davidovna". In: Os mais belos contos russos dos mais famosos autores. $2^{\mathrm{a}}$. série. Trad. Carlos Casanova. Rio de Janeiro: Vecchi, 1945.

O duelo. Trad. Paulo Corrêa Lopes. Porto Alegre: Globo, 1933.

"O ultraje". In: Os russos: antigos e modernos. Trad. Guilherme de Figueiredo. Coleção Contos do Mundo. Rio de Janeiro: Leitura, 1944.

“Um mártir da moda”. In: Os colossos do conto da velha e da nova Rússia. Trad. Alfredo Ferreira. Rio de Janeiro: Mundo Latino, 1944.

Vera. 1931.9

Yama (o bordel). Trad. Elias Davidovitch. Rio de Janeiro: Guanabara, 1935.

\section{Lavrenev, Boris:}

O sétimo camarada - novela russa. Trad. Jorge Amado [vide nota 3]. Coleção Ontem e Hoje, vol. 11. São Paulo: Brasiliense, 1945.

Vento. Trad. Jorge Amado [idem]. Coleção Ontem e Hoje, vol. 7. São Paulo: Brasiliense, 1945.

\footnotetext{
${ }^{9}$ Uma nota no jornal Diário Carioca de 7 de julho de 1931 informa o lançamento desse romance, sem menção à editora ou ao tradutor. Não localizei maiores informações.
} 


\section{Lebedinsky, E.}

A semana: novella. Trad. port. Cássio M. Fonseca. São Paulo: Pax, 1932.

\section{Leonov, Leonid:}

“A desventura de Ivan”. In: Os mais belos contos russos dos mais famosos autores. $2^{\mathrm{a}}$. série. Trad. Alfredo Ferreira. Rio de Janeiro: Vecchi, 1945.

\section{Lérmontov, Mikhail:}

"Ashik-Kerib (Um conto de fadas turco)". In: Os colossos do conto da velha e da nova Rússia. Trad. J. da Cunha Borges. Rio de Janeiro: Mundo Latino, 1944.

"O fatalista". In: Os russos: antigos e modernos. Trad. José Lins do Rego. Coleção Contos do Mundo. Rio de Janeiro: Leitura, 1944.

Leskov, Nikolai S. (Nicolau, Lescov, Lieskov):

"Lady Macbeth de Mtsenk". In: Os colossos do conto da velha e da nova Rússia. Trad. J. da Cunha Borges. Rio de Janeiro: Mundo Latino, 1944.

"Um tolo". In: Os russos: antigos e modernos. Trad. Joel Silveira. Coleção Contos do Mundo. Rio de Janeiro: Leitura, 1944.

Merejkóvski, Dmitri (Dimitri, Dmitry, [de] Merejkowski):

A morte dos deuses - Romance de Juliano o Apóstata. Trad. J. Ferreira-Souza Ferreira. Rio de Janeiro: Garnier, $1902 .^{10}$

Jesus desconhecido. Trad. Gustavo Barroso. São Paulo: Nacional, 1935.

Juliano, o apóstata (A morte dos deuses). Trad. Paulo Moreira da Silva. Coleção Grandes Biografias. Porto Alegre: Globo, 1945.

Napoleão - o homem e sua vida. Trad. Agrippino Grieco. São Paulo: Nacional, 1934.

O romance de Leonardo da Vinci (A ressurreição dos deuses). Trad. Brenno Silveira. Porto Alegre: Globo, 1946.

Tutankhamon em Creta (O nascimento dos deuses). Trad. "E.D.A.”. Rio de Janeiro: Calvino, 1934.

\footnotetext{
${ }^{10}$ Desde o começo do século passado, a H. Garnier passou a publicar diversas traduções portuguesas com créditos espúrios. Valeria a pena comparar essa "traducção brazileira", como vem estampado na página de rosto, à do lusitano Anibal de Vasconcelos (Porto: Moreira, 1901).
} 
“Um quadro de história". In: Os mais belos contos russos dos mais famosos autores. $1^{\mathrm{a}}$. Série. Trad. Manuel R. da Silva. Rio de Janeiro: Vecchi, 1945.

\section{Nemirovsky, Irène:}

“A partida para a festa". In: Os mais belos contos russos dos mais famosos autores. $2^{\mathrm{a}}$. série. Trad. Edison Carneiro. Rio de Janeiro: Vecchi, 1945.

Nevierof, Aleksandr (Alexandre, Alexander, Newerof):

A cidade da fartura. Epopéia de um menino russo da geração atual. Trad. Jorge Amado [vide nota 3]. Coleção Ontem e Hoje, vol. 6. São Paulo: Brasiliense, 1945.

"Fome”. In: Contos soviéticos. Os novos da Rússia. Trad. Gabriel Marques. Collecção Literatura Moderna. São Paulo: Cultura Brasileira, c.1934. Reed. in: Contos soviéticos. Rio de Janeiro: O Cruzeiro, 1944.

Ognev, Nicolai (Nicolau, Ognief):

O diário de Costia Riabtsev. Trad. Jorge Amado [vide nota 3]. Coleção Ontem e Hoje, vol. 8. São Paulo: Brasiliense, 1945.

“Os favoritos”. In: Contos soviéticos. Os novos da Rússia. Trad. Gabriel Marques. Collecção Literatura Moderna. São Paulo: Cultura Brasileira, c.1934. Reed. in: Contos soviéticos. Rio de Janeiro: O Cruzeiro, 1944.

\section{Olencha, Iúri (Ioury):}

"O caroço de cereja". In: Os russos: antigos e modernos. Trad. Afonso Arinos de Melo Franco Sobrinho. Coleção Contos do Mundo. Rio de Janeiro: Leitura, 1944.

\section{Panferov, Fiodor:}

“A enfermeira". In: Os mais belos contos russos dos mais famosos autores. 2a série. Trad. Alfredo Ferreira. Rio de Janeiro: Vecchi, 1945.

\section{Pilniak, Boris:}

"Mãos sujas de terra". In: Contos soviéticos. Os novos da Rússia. Trad. Gabriel Marques. Collecção Literatura Moderna. São Paulo: Cultura Brasileira, c.1934. Reed. in: Contos soviéticos. Rio de Janeiro: O Cruzeiro, 1944. 
“O vento humano". In: Os mais belos contos russos dos mais famosos autores. $2^{\mathrm{a}}$. série. Trad. Alfredo Ferreira. Rio de Janeiro: Vecchi, 1945.

O Volga desemboca no Mar Cáspio. Trad. Dom José Paulo da Câmara. São Paulo: Cultura Brasileira, c.1934. Reed. Rio de Janeiro: O Cruzeiro, 1943.

Poliakhov, Aleksandr (Alexander, Poliakov):

Os russos não se rendem. Trad. Augusto Rodrigues e George Reizman. Rio de Janeiro: Panamericana, 1945.

“Tanks” em ação. Trad. Celso Garcia. Coleção As Grandes Reportagens de Guerra. Rio de Janeiro: Horizonte, 1945.

“Troféu”. In: Os mais belos contos russos dos mais famosos autores. $2^{\mathrm{a}}$. série. Trad. Alfredo Ferreira. Rio de Janeiro: Vecchi, 1945.

\section{Prishvin, Mikhail:}

"Um lobisomem na estepe". In: Os mais belos contos russos dos mais famosos autores. $2^{\mathrm{a}}$. série. Trad. Alfredo Ferreira. Rio de Janeiro: Vecchi, 1945.

Púchkin, Aleksandr (Alexandre, Alexander; Pouskine, Pushkin, Pushkine, Puschkin): “A camponesa disfarçada". In: Os mais belos contos russos dos mais famosos autores. $1^{\text {a }}$. Série. Trad. Manuel R. da Silva. Rio de Janeiro: Vecchi, 1945.

“A dama de espadas”. In: Os russos: antigos e modernos. Trad. Dias da Costa. Coleção Contos do Mundo. Rio de Janeiro: Leitura, 1944.

A dama de espadas. Trad. Álvaro Moreyra. Rio de Janeiro: Confraria de Bibliófilos Brasileiros Cattleya Alba, 1944 [edição restrita]. Reed. Rio de Janeiro: Brasilia Aeterna, 1945.

A filha do capitão. Trad. Paulo Corrêa Lopes. Porto Alegre: Globo, 1933.

A filha do capitão. Trad. Boris Solomonov (pseud. de Boris Schnaiderman). Coleção Os Maiores Êxitos da Tela. Rio de Janeiro: Vecchi, 1949.

"A tempestade de neve". In: Os colossos do conto da velha e da nova Rússia. Trad. Edison Carneiro. Rio de Janeiro: Mundo Latino, 1944.

Águia negra. Trad. anôn. Collecções Econômicas SIP, vol. 32. Rio de Janeiro: Civilização Brasileira, 1935. ${ }^{11}$

\footnotetext{
${ }^{11}$ Para a adoção do título Águia negra para o título original Dubróvski, sem dúvida foi decisivo o grande sucesso do filme estadunidense The Eagle (1925), estrelado por Rodolfo Valentino no papel do Águia
} 
Águia negra, A dama de espadas, Um tiro. Trad. Cira Neri. Rio de Janeiro: Pongetti, 1937.

Águia negra. Contém: "Águia negra”, "O negro de Pedro, o Grande”, "O encarregado da estação" e "Kirdjali". Trad. Boris Solomonov (pseud. de Boris Schnaiderman). Coleção Os Maiores Êxitos da Tela. Rio de Janeiro: Vecchi, 1949.

"O chefe de posta". In: Os russos: antigos e modernos. Trad. Aníbal Machado. Coleção Contos do Mundo. Rio de Janeiro: Leitura, 1944.

"Um disparo memorável”. In: Os mais belos contos russos dos mais famosos autores. $2^{\text {a }}$. série. Trad. Carlos Casanova. Rio de Janeiro: Vecchi, 1945.

\section{Rachmanova, Alia:}

A fábrica do novo homem. Trad. Felipa Muniz. Porto Alegre: Globo, 1937.

Casamentos na tormenta vermelha. Trad. Felipa Muniz. Porto Alegre: Globo, 1940.

Diário duma exilada russa. Trad. Esther de Viveiros. Coleção Nobel. Porto Alegre: Globo, 1939.

Estudantes, amor, Tscheka e morte. Trad. Felipa Muniz. Coleção Nobel. Porto Alegre: Globo, 1936.

\section{Romanov, Panteleimon (Romanof):}

“Trevas”. In: Contos soviéticos. Os novos da Rússia. Trad. Gabriel Marques. Collecção Literatura Moderna. São Paulo: Cultura Brasileira, c.1934. Reed. in: Contos soviéticos. Rio de Janeiro: O Cruzeiro, 1944.

\section{Savitsch, Ovadi:}

"Os ladrões de cavallo". In: Contos soviéticos. Os novos da Rússia. Trad. Gabriel Marques. Collecção Literatura Moderna. São Paulo: Cultura Brasileira, c.1934. Reed. in: Contos soviéticos. Rio de Janeiro: O Cruzeiro, 1944.

\section{Schapiro, Leon:}

"O homem da cruz". In: Os mais belos contos russos dos mais famosos autores. $2^{\mathrm{a}}$. série. Trad. Gilberto Galvão. Rio de Janeiro: Vecchi, 1945.

Negra, lançado no Brasil em 1926. Isso sem contar o sucesso do italiano Aquila Nera (direção de Riccardo Freda, 1946), que se reflete no título da Vecchi em 1949, em sua coleção "Os Maiores Êxitos da Tela". 
Seifullin, Lydia (Lídia):

"Mãe". In: Os russos: antigos e modernos. Trad. Caio de Freitas. Coleção Contos do Mundo. Rio de Janeiro: Leitura, 1944.

Semionov, Serguei (Sergio, Semionof):

“O nascimento do escravo". In: Contos soviéticos. Os novos da Rússia. Trad. Gabriel Marques. Collecção Literatura Moderna. São Paulo: Cultura Brasileira, c.1934. Reed. in: Contos soviéticos. Rio de Janeiro: O Cruzeiro, 1944.

\section{Simonov, Konstantin:}

“A vantagem de ser valente". In: Os colossos do conto da velha e da nova Rússia. Trad. Manuel R. da Silva. Rio de Janeiro: Mundo Latino, 1944.

\section{Skhaginiam, Marieta:}

"A enfermeira Zedrik". In: Contos soviéticos. Os novos da Rússia. Trad. Gabriel Marques. Collecção Literatura Moderna. São Paulo: Cultura Brasileira, c.1934. Reed. in: Contos soviéticos. Rio de Janeiro: O Cruzeiro, 1944.

\section{Sologub, Féodor:}

“O arco". In: Os russos: antigos e modernos. Trad. Luís Jardim. Coleção Contos do Mundo. Rio de Janeiro: Leitura, 1944.

\section{Sosulia, Jefim:}

“O direito à vida”. In: Os mais belos contos russos dos mais famosos autores. 1ª Série. Trad. Manuel R. da Silva. Rio de Janeiro: Vecchi, 1945.

\section{Surguchov, Ilia Dmitrievitch (J.):}

"Os pais". In: Os mais belos contos russos dos mais famosos autores. 1'. Série. Trad. Marina Salles Goulart de Andrade. Rio de Janeiro: Vecchi, 1945.

\section{Tasin, Nikolai:}

"Nas garras da morte". In: Os mais belos contos russos dos mais famosos autores. $1^{\text {a }}$. Série. Trad. Manuel R. da Silva. Rio de Janeiro: Vecchi, 1945. 
Tchekhov, Anton (Tchecoff, Tchecov, Tschecov, Chejov):

“A linguaruda". In: Antologia dos grandes contos humorísticos. Trad. anôn. São Paulo: Brasiliense, 1944.

Amor impossível. Contém: "Amor impossível”, "O bilhete premiado", "No restaurante postal", "Um animal de raça", "O enxoval". Trad. Marina Salles Goulart de Andrade e Gilberto Galvão. Coleção Os Grandes Nomes. Rio de Janeiro: Vecchi, 1945.

Contos. Contém: "La cigale", "Sonhos", "O buraco", “O beijo", "Varka" e "A estepe". Trad. Costa Neves. Rio de Janeiro: W. M. Jackson, 1950.

"Do amor". In: Os mais belos contos russos dos mais famosos autores. $2^{\text {a }}$. série. Trad. Edison Carneiro. Rio de Janeiro: Vecchi, 1945.

"No mar da Criméia". In: As obras-primas do conto universal. Trad. Almiro R. Barbosa e Edgard Cavalheiro. Porto Alegre: Martins, 1943.

“O coração de Olenka”. In: Os russos: antigos e modernos. Trad. Rachel de Queiroz. Coleção Contos do Mundo. Rio de Janeiro: Leitura, 1944.

"O homem do estojo". In: Os colossos do conto da velha e da nova Rússia. Trad. Edison Carneiro. Rio de Janeiro: Mundo Latino, 1944.

“O inimigo". In: Os russos: antigos e modernos. Trad. Oswaldo Alves. Coleção Contos do Mundo. Rio de Janeiro: Leitura, 1944.

O pavilhão $n^{o}$. 6. Contém: "O pavilhão no. 6", "A desgraça", "A felicidade”, “A obra de arte" e "Os simuladores". Trad. anôn. [Georges Selzoff e F. Olandim]. Bibliotheca dos Auctores Russos. São Paulo: Cultura, 1931.

“O violino de Rothschild”. In: Os russos: antigos e modernos. Trad. Luís Martins. Coleção Contos do Mundo. Rio de Janeiro: Leitura, 1944.

Olhos mortos de sono. Contém: "Olhos mortos de sono", "Os nervos", "A condecoração", "Medidas preventivas", “A vingança”, "O orador”, “As sensações fortes", "Um bom final”, “A obra de arte", "Uma noite de terror", "História de uma enguia”, “O medo”, “Entre meninos”, “A jóia roubada”, “Dois valentes”, “O vingador”, "Ivan Matveievitch", "O álbum”, "Língua comprida”, "Grischa”, "Celebridades”, "Na hospedaria”, “Os veranistas”. Trad. Carlos M. A. Bittencourt. Coleção Seleta, vol. 2. São Paulo: Assunção, 1945.

Os inimigos. Contém: "Os inimigos", "Delírio (Gussieff)", "Algazarra em família", "No carro (o caminho da mestra-escola)", "Verotchka", "Estudante (conto do jardineiro 
chefe)", "Zinotchka" e "Uma noite atroz". Trad. anôn. [Georges Selzoff e F. Olandim]. Bibliotheca dos Auctores Russos. São Paulo: Cultura, 1930.

“Os ladrões”. In: 3 novelas russas. Trad. José de Barros Pinto. São Paulo: Flama, 1944.

"Vanka". In: Os russos: antigos e modernos. Trad. Manuel Bandeira. Coleção Contos do Mundo. Rio de Janeiro: Leitura, 1944.

Um caso de clínica médica. Trad. Yolanda Vettori. Coleção As 100 Obras-primas da Literatura Universal. Rio de Janeiro: Pongetti, 1945.

“Uma boa mulher”. In: Os mais belos contos russos dos mais famosos autores. 1". Série. Trad. Marina Salles Goulart de Andrade. Rio de Janeiro: Vecchi, 1945.

"Uma frase milagrosa". In: Os mais belos contos burlescos, irônicos e sarcásticos, dos mais famosos autores. Trad. Alfredo Ferreira. Rio de Janeiro: Vecchi, 1947.

Teffi, Nadezhda (Nadine, Teffy):

“Cartas de amor”. In: Contos soviéticos. Trad. Luiz Alípio de Barros. Rio de Janeiro: O Cruzeiro, 1944.

"Estratégia no vagão". In: Os mais belos contos russos dos mais famosos autores. $2^{\mathrm{a}}$. série. Trad. J. da Cunha Borges. Rio de Janeiro: Vecchi, 1945.

"O faquir". In: Os mais belos contos russos dos mais famosos autores. $1^{\text {a }}$. Série. Trad. Frederico dos Reys Coutinho. Rio de Janeiro: Vecchi, 1945.

Tieliechóv, Nikolai (Nicolau, Telechov):

"A miséria". In: Os mais belos contos russos dos mais famosos autores. 2a . série. Trad. Alfredo Ferreira. Rio de Janeiro: Vecchi, $1945{ }^{12}$

“O duelo". In: Os russos: antigos e modernos. Trad. Wilson Velloso. Coleção Contos do Mundo. Rio de Janeiro: Leitura, 1944.

Tikhonov, Nikolai (Nicolau):

"O califa". In: Os russos: antigos e modernos. Trad. Clóvis Ramalhete. Coleção Contos do Mundo. Rio de Janeiro: Leitura, 1944.

“Um novo ser". In: Os colossos do conto da velha e da nova Rússia. Trad. Manuel R. da Silva. Rio de Janeiro: Mundo Latino, 1944.

\footnotetext{
${ }^{12}$ Grafado nessa edição da Vecchi como Felekhov. Agradeço a correta identificação a Bruno Gomide, cujo estudo, aliás, mostra que este mesmo conto fora publicado na revista Careta em 1917, já com a grafia equivocada de Telekhov (GOMIDE, 2004, 179 e 487).
} 


\section{Tikhonov, Vladimir:}

“O lobo do mar". In: Os russos: antigos e modernos. Trad. Evandro Pequeno. Coleção Contos do Mundo. Rio de Janeiro: Leitura, 1944.

"Paciência". In: Os russos: antigos e modernos. Trad. Evandro Pequeno. Coleção Contos do Mundo. Rio de Janeiro: Leitura, 1944.

\section{Tolstói, Alexis (Alexei):}

A cultura soviética. Trad. Paim Júnior. Rio de Janeiro: Vitória, 1945.

O soviet em Marte. Trad. anôn. Rio de Janeiro: Calvino, 1933.

“Os vurdalaks". In: Os mais belos contos russos dos mais famosos autores. $2^{\mathrm{a}}$. série. Trad. Alfredo Ferreira. Rio de Janeiro: Vecchi, 1945.

Pedro, o Grande. Coleção Documentos Humanos. Rio de Janeiro: Zélio Valverde, 1945.

“Um relato de Ivã Sudariev". In: Os colossos do conto da velha e da nova Rússia. Trad. Manuel R. da Silva. Rio de Janeiro: Mundo Latino, 1944.

Tolstói, Lev (Leon, Leão, Liev, Tolstoy):

A escravidão moderna. Trad. anôn. Collecções Econômicas SIP, Vol. 50. Rio de Janeiro: Civilização Brasileira, 1937.

"A morte de Ivan Ilitch". In: Os russos: antigos e modernos. Trad. Carlos Lacerda. Coleção Contos do Mundo. Rio de Janeiro: Leitura, 1944.

A morte de Ivan Ilitch e Amo e servidor. Trad. Gulnara Lobato de Morais. São Paulo: Saraiva, 1948.

A palavra de Jesus. Trad. anôn. Rio de Janeiro: H. Antunes, 1931.

A sonata a Kreutzer. Trad. anôn. Rio de Janeiro: J. Ribeiro dos Santos, 1905.

A sonata a Kreutzer. Trad. anôn. Rio de Janeiro: Empreza Romantica, 1909.

A sonata a Kreutzer. Trad. Amando Fontes. Coleção Fogos Cruzados. Rio de Janeiro: José Olympio, 1941.

A sonata a Kreutzer (romance). Trad. Vicente Vaz. Rio de Janeiro: Miniatura, 1948.

A sonata de Kreutzer. Trad. anôn. São Paulo: Teixeira, 1913.

A tortura da carne. Trad. anôn. Rio de Janeiro: Civilização Brasileira, c.1936.

A tortura da carne/ De onde viria o castigo? Trad. anôn. Edições Colête. São Paulo: A Bolsa do Livro, 1945. 
A verdadeira vida. Trad. Rossini Tavares de Lima. Coleção Os Grandes Pensadores. Rio de Janeiro: Vecchi, 1947.

"Alexis - o 'pote"”. In: Os russos: antigos e modernos. Trad. Joracy Camargo. Coleção Contos do Mundo. Rio de Janeiro: Leitura, 1944.

Amo e creado. Trad. “A. F.”. Rio de Janeiro: João do Rio, 1926.

Ana Karênina. Trad. Lúcio Cardoso. Coleção Fogos Cruzados. Rio de Janeiro: José Olympio, 1943.

Ana Karenina. Trad. revista por Marques Rebelo. Rio de Janeiro: Pongetti, 1943.

Anna Karenine. Trad. anôn. São Paulo: Sociedade Impressora Paulista, 1930. Reed. São Paulo: Nacional, 1930.

“As três palavras divinas". In: As obras-primas do conto universal. Trad. Almiro R. Barbosa e Edgard Cavalheiro. Porto Alegre: Martins, 1943.

“De onde viria o castigo?”. In: Os colossos do conto da velha e da nova Rússia. Trad. José Dauster. Rio de Janeiro: Mundo Latino, 1944.

Diários íntimos (com Sofia Tolstoi). Trad. Frederico dos Reys Coutinho. Rio de Janeiro: Vecchi, 1943.

Duas novelas: O príncipe Kassatsky e O diabo. Trad. Caio Jardim. Rio de Janeiro: Universitária, 1940.

"Francisca". In: Os mais belos contos russos dos mais famosos autores. 1". Série. Trad. Marina Salles Goulart de Andrade. Rio de Janeiro: Vecchi, 1945.

Guerra e paz. Trad. Gustavo Nonnenberg. Coleção Biblioteca dos Séculos. Porto Alegre: Globo, 1942.

Homens e escravos. Trad. Cira Neri. Coleção As 100 Obras-Primas da Literatura Universal. Rio de Janeiro: Pongetti, 1943.

"Ivan, o imbecil". In: Três novelas russas. Trad. Lúcio Cardoso. Rio de Janeiro: A Noite, 1947.

Katia. Trad. Lêdo Ivo. Rio de Janeiro: Panamericana, c.1944.

Khadji-Murat. Trad. anôn. [Georges Selzoff e Allyrio M. Wanderley]. Bibliotheca de Auctores Russos. São Paulo: Cultura, 1931.

"Khadji-Murat". In: Três novelas russas. "Tradução revista pelo departamento editorial" [versão adulterada da tradução de Selzoff/ Wanderley, Cultura, 1931]. Coleção Grandes Romances Universais. São Paulo: W. M. Jackson, 1947.

Memórias (infância, adolescência e juventude). Trad. Rachel de Queiroz. Coleção Memórias, Diários, Confissões. Rio de Janeiro: José Olympio, 1944. 
O canto do cysne. Trad. anôn. Collecções Econômicas SIP, vol. 59. Rio de Janeiro: Civilização Brasileira, 1937.

O diabo branco (Khadji-Murat). Trad. António Sérgio. Rio de Janeiro: Civilização Brasileira, 1934.

O diabo branco. "Tradução revista" [versão levemente adulterada da tradução de Selzoff/Wanderley, Cultura, 1931]. São Paulo: Publicações Brasil, c. 1944.

O diabo branco. Trad. Boris Solomonov [pseud. de Boris Schnaiderman]. Coleção Os maiores êxitos da tela. Rio de Janeiro: Vecchi, 1949.

O drama do padre Sérgio. Trad. anôn. São Paulo: Nosso Livro, c.1944.

O que eu penso da guerra. Trad. anôn. Rio de Janeiro: H. Antunes, 1909.

O quinhão da mulher, impressionante relato da própria heroína. Trad. João Cabral. Rio de Janeiro: Brasilica, 1940.

O trabalho (com Timoteo Bondareff). Trad. João Cabral. Rio de Janeiro: Marisa, 1934.

Os cossacos. Trad. Sérgio Azevedo. Rio de Janeiro: Livraria Marisa, 1931.

Os cossacos. Trad. anôn. São Paulo: Sociedade Impressora Paulista, 1932. Reed. em Collecções Econômicas SIP, vol. 4. Rio de Janeiro: Civilização Brasileira, 1934.

Os cossacos. Trad. Almir de Andrade. Coleção Fogos Cruzados. Rio de Janeiro: José Olympio, 1942.

Os cossacos. Trad. anôn. São Paulo: Clube do Livro, 1944.

Os martyres do dinheiro [Na floresta - novela (narrativa de um yunker) - 1854-1855]. Trad. anôn. Collecções Econômicas SIP, vol. 56. Rio de Janeiro: Civilização Brasileira, 1937.

“Os três staretzi”. In: Os russos: antigos e modernos. Trad. Alfredo Mesquita. Coleção Contos do Mundo. Rio de Janeiro: Leitura, 1944.

Padre Sergio. Trad. anôn. [Georges Selzoff e Allyrio M. Wanderley]. Bibliotheca de Auctores Russos. São Paulo: Cultura, 1931.

Polikuchka. Trad. Henrique Cordeiro. Rio de Janeiro: Vitória, 1944.

Resurreição, romance celebre. Trad. Carlos Cintra. Collecção de Obras Celebres. Rio de Janeiro: Americana, 1931. Reed. Rio de Janeiro: Guanabara, 1935.

Ressurreição. Trad. anôn. Rio de Janeiro: Civilização Brasileira, 1936.

Ressurreição. Trad. Waldemar Cavalcanti. Coleção Fogos Cruzados. Rio de Janeiro: José Olympio, 1944.

Ressurreição. Trad. revista por Marina Salles Goulart de Andrade. Rio de Janeiro: Cia. Brasil, s/d [c. 1945]. 
Sebastopol. Trad. F. J. da Silva Ramos. Coleção Excelsior. São Paulo: Martins, 1944. Senhor e servo. Trad. anôn. Coleção Azul. Rio de Janeiro: Aurora, c.1946.

Sonata de Kreutzer. Trad. anôn. São Paulo: Sociedade Impressora Paulista, c.1930.

Três novelas da Rússia. Contém: "O violinista Alberto", "Um animal como poucos" e “Romance inacabado”. Trad. anôn. São Paulo: Nosso Livro, 1944.

Turguêniev, Ivan (Ivã, Turgenev, Turgenov, Turguenev, Turguenieff):

"A aventura do tenente Yergunov". In: Os mais belos contos russos dos mais famosos autores. 2 ${ }^{\mathrm{a}}$. série. Trad. Galvão de Queiroz. Rio de Janeiro: Vecchi, 1945.

Águas da primavera. Trad. Brito Broca e Georges Selzoff. Bibliotheca de Auctores Russos. São Paulo: Cultura, 1932.

Ássia. Trad. anôn. Coleção Volga. São Paulo: Unitas, c.1934.

“Ássia”. In: Contos russos. Trad. anôn. Edições Colête, vol. 7. São Paulo: A Bolsa do Livro, 1944.

"Biriuk". In: Os mais belos contos russos dos mais famosos autores. 1'. Série. Trad. Manuel R. da Silva. Rio de Janeiro: Vecchi, 1945.

Fumaça. Trad. Jorge Moreira Nunes. Rio de Janeiro: Pongetti, 1945.

Ninho de fidalgos. Trad. Elsie Lessa e Georges Selzoff. Bibliotheca de Auctores Russos. São Paulo: Cultura, 1932.

"O encontro". In: Os russos: antigos e modernos. Trad. Lauro Escorel. Coleção Contos do Mundo. Rio de Janeiro: Leitura, 1944.

"O médico do distrito". In: Os colossos do conto da velha e da nova Rússia. Trad. Frederico dos Reys Coutinho. Rio de Janeiro: Mundo Latino, 1944.

O primeiro amor, O medo, Birouck. Trad. Brito Broca. São Paulo: José Olympio, 1949. Paes e filhos. Trad. Ivan Emilianovitch. São Paulo: Cultura Brasileira, 1935. Reed. São Paulo: Martins, 1941. Reed. Pais e filhos. São Paulo: Clube do Livro, 1943.

Primeiro Amor e Assia. Trad. Edy Maria Dutra da Costa. São Paulo: Assunção, 1946.

Roudine. Trad. Elias Davidovitch. Collecção Benjamin Costallat. Rio de Janeiro: Flores e Mano, 1932.

Rudine. Trad. revista por Marques Rebelo. Rio de Janeiro: Pongetti, 1943.

Rudine. Trad. anôn. São Paulo: Clube do Livro, 1947.

Sinaida. Trad. anôn. Coleção Azul. Rio de Janeiro: Aurora, c.1946.

Terra virgem. Trad. Jorge Moreira Nunes. Rio de Janeiro: Panamericana, 1944. 
Um búlgaro - romance. Trad. anôn. Rio de Janeiro: Universal, 1933. ${ }^{13}$

Tzensky, Serguei S. (Sergei):

“O homem que não podia ser morto". In: Os mais belos contos russos dos mais famosos autores. $2^{\text {a }}$. série. Trad. Alfredo Ferreira. Rio de Janeiro: Vecchi, 1945.

\section{Uspenski, Nicolau V.:}

"O mestre-escola de aldeia”. In: Os russos: antigos e modernos. Trad. Marques Rebelo. Coleção Contos do Mundo. Rio de Janeiro: Leitura, 1944.

Vassilevska, Vanda (Wanda, Wasilewska):

O arco-íris. Trad. Emaragdo Marroquim e Valdemar Cavalcanti. Rio de Janeiro: O Cruzeiro, 1945.

Vieressaiev, V. (Veressaief, pseud. de Vikenti V. Smidovitch):

Beco sem sahida (novella russa). Trad. Alexandre Wainstein e Galeão Coutinho. São Paulo: Pax, 1931.

\section{Vinnitchenko, Vladimir:}

"Ao sopro dos ventos, dos ventos furiosos". In: Os russos: antigos e modernos. Trad. Álvaro Moreyra. Coleção Contos do Mundo. Rio de Janeiro: Leitura, 1944.

Virta, Nikolai E. (pseud. de Nikolai Karelsky):

Solidão. Trad. Jorge Amado [vide nota 3]. Coleção Ontem e Hoje, vol. 9. São Paulo: Brasiliense, 1945.

\section{Zamiátin, Euguêni (Eugênio):}

“A caverna". In: Os russos: antigos e modernos. Trad. Lúcio Cardoso. Coleção Contos do Mundo. Rio de Janeiro: Leitura, 1944.

\footnotetext{
${ }^{13}$ Apenas a título de curiosidade, escapando ao nosso quadro temporal, vale notar que Um búlgaro, na tradução portuguesa de Lourenço Cayolla, foi uma das primeiras obras russas publicadas em livro no Brasil. Saiu em 1897 em nada menos que três editoras: pela Laemmert carioca e pelas pelotenses Echenique \& Irmão (Livraria Universal) e Americana (de Carlos Pinto). Não me parece improvável que essa tradução anônima lançada pela Livraria Universal carioca em 1933 retome a mesma tradução de Cayolla, mas não cheguei a cotejá-las.
} 
Zochtchenko, Mikhail (Zostchenko, Zóschenko):

“A maleta de lona". In: Os mais belos contos burlescos, irônicos e sarcásticos, dos mais famosos autores. Trad. Alfredo Ferreira. Rio de Janeiro: Vecchi, 1947.

No paraíso bolchevista (quadros da vida russa). Trad. Roman Poznanski. Rio de Janeiro: H. Antunes, 1929.

Sem autoria (contos tradicionais e folclóricos):

Os mais belos contos de fadas russos, $1^{\text {a }}$. série. Contém catorze contos: "Kotschei, o imortal”, "Sadko, o mercador", “O czar Saltan”, "O galo de ouro", "Ruslan e Ludmila", "Cirilo, o curtidor", "A fada que sabia tudo", "A princesa adormecida e os sete gigantes", “A princesa serpente”, "Como foram destruídos os Bogatirs na Santa Rússia" [todos estes em tradução de Galvão de Queiroz]; "O anel encantado", "O gênio da estepe", "Os dois filhos de Ivã, o soldado" e "O camponês prevenido" [estes em tradução de Manuel R. da Silva]. Rio de Janeiro: Vecchi, 1945.

Infelizmente, não cheguei a localizar o conteúdo de Os mais belos contos de fadas russos, $2^{\text {a }}$. série. Sei apenas que as traduções foram feitas por Dulce Cordeiro, Persiano da Fonseca e Alfredo Ferreira. Rio de Janeiro: Vecchi, 1945.

\section{Conclusão}

Os dados acima apresentados sugerem um painel que pode ser analisado sob vários pontos de vista. O mínimo que se pode dizer, à guisa de conclusão, é que a publicação de livros de literatura russa e soviética no Brasil começou a demonstrar algum vigor no Brasil a partir dos anos 1930. Por um lado, isso não admira muito, pois foi apenas naquela década que se registrou o efetivo arranque da indústria editorial entre nós. Por outro lado, é sugestivo notar que a publicação de obras russas e soviéticas no Brasil não esteve ausente desse processo, acompanhando-o de perto, ainda que a repressão da ditadura varguista em nada tenha colaborado, muito pelo contrário, para uma difusão que, provavelmente, teria um potencial bem maior já naquela época. Todos estes, e muitos outros, são temas que demandam estudos específicos, para os quais espero que este levantamento possa contribuir com dados concretos. 


\section{Agradecimentos}

Agradeço a Aline Veras, Anna Naldi (FBN), Bruno Gomide, Gutemberg de Medeiros, José Eisenberg, Rodrigo Alves do Nascimento, Saulo von Randow Jr., Simei Maoski e Tiago Pavan, por importantes informações biobibliográficas; e a Federico Carotti, por seu apoio constante, a quem dedico esta pesquisa.

\section{Fontes primárias:}

Fundação Biblioteca Nacional, catálogo antigo

Hemeroteca Digital da Fundação Biblioteca Nacional

Portal Estante Virtual

Vários acervos e arquivos digitais de bibliotecas, revistas e jornais

\section{Fontes secundárias:}

Amorim, Sônia Maria de. Em busca de um tempo perdido - Edição de literatura traduzida pela Editora Globo (1930-1950). São Paulo: EdUSP, Com-Arte; Porto Alegre: Ed. UFRGS, 1999.

Bottmann, Denise. "Bibliografia de Tolstói no Brasil”. In: Bartlett, Rosamund. Tolstói, a biografia. São Paulo: Biblioteca Azul, 2013, 587-96.

Bottmann, Denise. "Georges Selzoff, uma crônica". In: Tradução em Revista 14, 2013/1, 208-229. Disponível em <http://www.maxwell.lambda.ele.pucrio.br/trad_em_revista.php?strSecao=input0>

Cardoso, Rafael. “O início do design de livros no Brasil”. In: Cardoso, Rafael (org.), $O$ design brasileiro antes do design. São Paulo: Cosac Naify, 2005, 160-96.

Gomide, Bruno. Da estepe à caatinga: o romance russo no Brasil (1887-1936). Tese de doutorado. IEL/UNICAMP, Campinas: 2004. São Paulo: EdUSP, 2011.

Hallewell, Laurence. O livro no Brasil - sua história. Trad. Maria da Penha Villalobos, Lólio Lourenço de Oliveira e Geraldo Gerson de Souza. 2a . ed. rev. e ampl. São Paulo: EdUSP, 2005.

Karepovs, Dainis. Bücher - Uma amostra do design gráfico editorial de esquerda no Brasil dos anos 1920 aos anos 1970. Disponível em 
<https://www.facebook.com/dakar.karepovs/media_set?set=a.105221482988834.9963.1 $\underline{00005029474297 \& \text { type }=3>}$

Medeiros, Gutemberg de. "Entrevista com Boris Solomônovitch Schnaiderman”. In: Revista USP 75, set./nov. 2007.

Nascimento, Rodrigo Alves do. Tchékhov no Brasil: A construção de uma atualidade.

Dissertação de mestrado. FFLCH/USP, São Paulo: 2013. Disponível em 〈http://www.teses.usp.br/teses/disponiveis/8/8155/tde-05062013-115641/pt-br.php〉

Ninitch, Zoran. “A obra literária do autor”. In: Saudade - A epopeia de uma palavra. Rio de Janeiro: Rodrigues \& Cia., 1939, 43-47.

Palamartchuk, Ana Paula. "Intelectuais, esquerdas e cultura no Brasil: os anos 1930". In: Roxo, Marcos, e Sacramento, Igor (orgs.): Intelectuais partidos: os comunistas e as mídias no Brasil. Rio de Janeiro: E-papers, 2012, 43-72.

Encontram-se outros materiais adicionais, em especial sobre as traduções brasileiras de Dostoiévski, Púchkin e Tolstói até a data presente, em <http://naogostodeplagio.blogspot.com.br/search/label/russos\%20no\%20brasil > 